\title{
The Role of Magnetic Fields in Star Formation
}

\author{
Ralph E. Pudritz ${ }^{1,2}$, Mikhail Klassen ${ }^{1}$, Helen Kirk ${ }^{1,3}$, Daniel Seifried ${ }^{4}$ \\ and Robi Banerjee ${ }^{4}$ \\ ${ }^{1}$ Department of Physics and Astronomy, McMaster University, \\ Hamilton, ON L8S 4M1, Canada \\ ${ }^{2}$ Origins Institute, McMaster University, Hamilton, ON L8S 4M1, Canada \\ email: pudritz@mcmaster.ca \\ ${ }^{3}$ Banting Fellow, McMaster University; now at Radio Astronomy Program, NRC, Canada \\ ${ }^{4}$ Hamburg Sternwarte, University of Hamburg, \\ Gojenbergsweg 11221209 Hamburg - Germany
}

\begin{abstract}
Stars are born in turbulent, magnetized filamentary molecular clouds, typically as members of star clusters. Several remarkable technical advances enable observations of magnetic structure and field strengths across many physical scales, from galactic scales on which giant molecular clouds (GMCs) are assembled, down to the surfaces of magnetized accreting young stars. These are shedding new light on the role of magnetic fields in star formation. Magnetic fields affect the gravitational fragmentation and formation of filamentary molecular clouds, the formation and fragmentation of magnetized disks, and finally to the shedding of excess angular momentum in jets and outflows from both the disks and young stars. Magnetic fields play a particularly important role in angular momentum transport on all of these scales. Numerical simulations have provided an important tool for tracking the complex process of the collapse and evolution of protostellar gas since several competing physical processes are at play - turbulence, gravity, MHD, and radiation fields. This paper focuses on the role of magnetic fields in three crucial regimes of star formation: the formation of star clusters emphasizing fragmentation, disk formation and the origin of early jets and outflows, to processes that control the spin evolution of young stars.
\end{abstract}

Keywords. magnetic fields, star formation, filaments, molecular clouds, protostellar disks, magnetic braking, outflows, protostellar rotation.

\section{Introduction}

The theory of stellar structure and evolution informs us that the mass, rotation, and composition of stars control their fates. Two of these, the distribution of initial stellar masses (the initial mass function, or IMF) and the distribution of initial protostellar rotation rates can in principle be directly affected by magnetic forces. While star formation involves the interplay of turbulence, gravity, radiation, and magnetic fields on a variety of scales, can magnetic fields significantly affect the nature of the distribution of stellar masses of newly formed stars? Can they control the rotation rates of stars as they arrive onto the zero-age main sequence? The short answer to both of these questions is yes. The subtlety lies in teasing out whether or not magnetic effects are significantly muted in the complex interplay of these physical processes.

The origin of the IMF is linked to the distribution of overdense regions in star forming clouds. This is clearly seen in Figure 1(a) where observations of the conditions leading to stellar birth in a low mass, star forming cloud (the Pipe Nebula) are shown. Dense knots of gas known as cores are distributed along a filamentary structure. The mass spectrum 
of these cores known as the (Dense) Core Mass Spectrum or CMF is compared with the IMF in Figure 1(b). The data shows that these distributions are similar in form, where masses can be well determined, except that the CMF is shifted to higher masses by a factor of roughly 3. (Alves et al. 2007) The results are similar to many other studies (Motte et al. 1997, Testi \& Sargent, Johnstone et al. 2000). The CMF has a peak at around $1 M_{\odot}$ (André et al. 2010) whereas the most abundant stars in the IMF occur at $\simeq 0.2 M_{\odot}$, The shape of IMF can be well fit by a lognormal distribution with a powerlaw tail at high masses (Chabrier 2003). In another example of a more evolved, star forming cloud known as the Aquila Rift, $85 \%$ of the mass of the cloud can be fit by a lognormal distribution, while 15\% falls inside the power-law tail (Andre et al. 2011, Schneider et al. 2013). Turbulence readily produces lognormal density distributions. A simple interpretation of the relation between the IMF and the CMF is that physical processes such as radiation from the central star, or hydromagnetic outflows manage to disperse typically $2 / 3$ of the gas in the original cores.

A huge effort over the last decade has measured the distribution of spins of over 5000 young stars (review, Bouvier et al. 2013). A key finding is that young, low mass (0.1 - 1 $\left.M_{\odot}\right)$ pre main sequence (PMS) stars that are a few Myr old span a broad range of stellar spins with most ranging from periods of 1 - 10 days. More than half of the T Tauri stars are slow rotators; for typical TTS stars with masses $M_{*}=0.5 M_{\odot}$, radii $R_{*}=2 R_{\odot}$, and a period of 8 days, the ratio $f$ of the observed spin period to the break up spin rate is $f<0.1$. These systems undergo vigorous accretion which should push the rotation rates to near break up within a few $10^{5} \mathrm{yr}$. Here too, magnetic forces in the form of spin-down torques may be playing a decisive role.

In this review, we descend from the large physical scales associated with magnetic fields in galaxies and molecular clouds, to accretion disks and outflows around young stars, down to the surfaces T-Tauri stars undergoing active magnetospheric accretion from their surrounding accretion disks. We trace some of the important roles that magnetic fields can play in star formation.

\section{Magnetic fields and Filamentary Molecular Clouds in Galaxies}

Magnetic fields in molecular clouds ultimately arise as a consequence of dynamo action in the large scale interstellar medium of galaxies. They can be traced by polarized
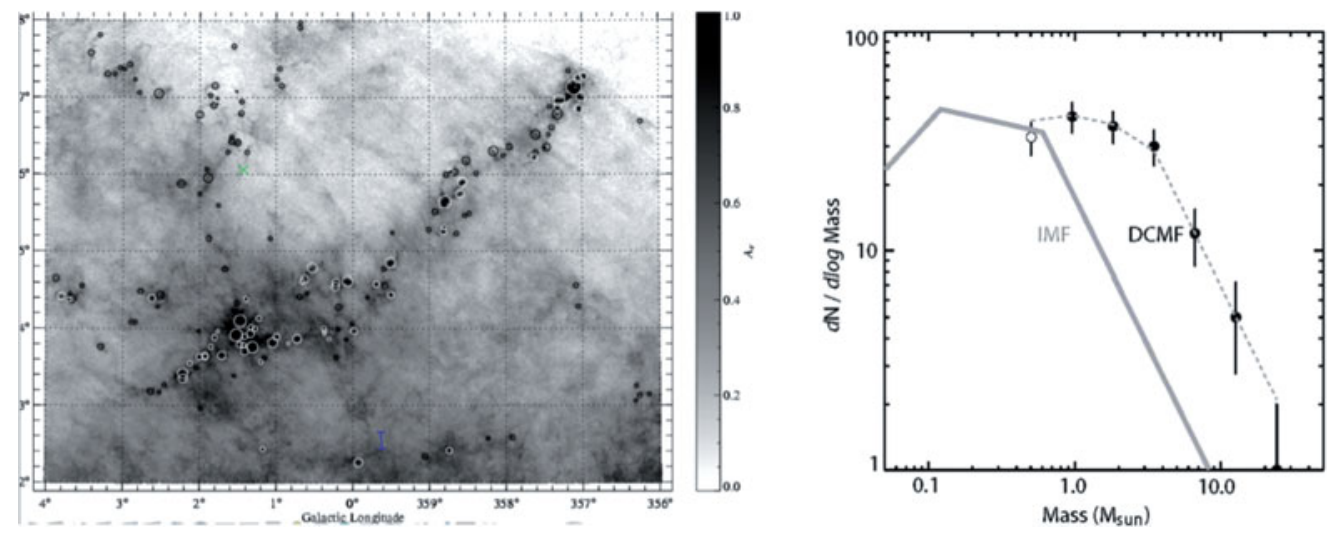

Figure 1. (a) Dense molecular cloud cores in the Pipe Nebula; (b) Comparison of the observed Core Mass Function (CMF) in the Pipe Nebular, describing the mass distribution of cores, with the Initial Mass Function which is the mass spectrum of newly formed stars. Adapted from Alves et al. (2007). 
synchrotron emission mapped by multifrequency radio observations. This allows the strength of the field perpendicular to the line of sight to be measured. The parallel component of the field is obtained by measuring the Faraday rotation, or rotation measure (RM) (review, Beck, 2012). Ordered fields with spiral arm geometry can be traced a large variety of galaxies (Beck 2005) and are strongest in the inter-arm region between optical spiral arms, with a strength of 10-15 $\mu G$ and are generated by galactic dynamos. In galaxies with strong spiral density waves, such as M51 shown in Figure 2(a), the field is compressed and sheared by the non-axisymmetric flows associated with the arm (Fletcher et al. 2011). The dominant dynamo mode is a quadrupolar type of field which includes a vertical component out of the galactic plane (Heeson et al. 2009). The distribution of field strengths shown in Figure 2(b) for several tens of disk galaxies is $B_{\text {tot }} \simeq 17 \pm 14 \mu G$ with a mean field of $B_{\text {mean }} \simeq 5 \pm 3 \mu G$. These measurements show that magnetic fields are in equipartition with energy in the cosmic rays and the interstellar medium on these scales.

These fields are subsequently incorporated into the formation of giant molecular clouds in galaxies. This may occur by several different mechanisms. Strong fields are buoyant as was first shown by Parker. In the self-gravitating gas of the galaxy, a combined ParkerJeans instability operates (Elmegreen 1982) wherein the gravity is the strongest in the galactic plane and the magnetic buoyancy peaks far above the plane. The growth time of such an instability in a galaxy is about the Jeans timescale or $\left(4 \pi G \rho_{o}\right)^{-1 / 2} \simeq 23 n_{o}^{1 / 2}$ million years where $n_{o}$ is the volume number density of the gas. Gas draining down the large scale buoyant magnetic loops rising through the galactic disk accumulates into magnetized $10^{6}$ solar mass GMCs in 10 Myr in this scenario. Recent work has emphasized the possible role of the magneto-rotational instability (MRI) operating together with the Toomre gravitational instability for galactic disks, in creating magnetized molecular clouds (Sellwood \& Balbus 1999, Kim et al. 2003). Since the orbital frequencies of gas in galaxies is a decreasing function of radius $\left(\Omega(r) \propto r^{-1}\right)$, they will be subject to the MRI instability which creates vigorous turbulence. In sufficiently dense parts of the galaxy, the Toomre instability will act like a swing amplifier acting on the larger fluctuations, capable of building molecular clouds up to $10^{7}$ solar masses. In either case, the magnetized gas in a galaxy is, together with gravity, a major participant in the creation of star forming GMCs.

Star formation is strongly linked to the structure of GMCs. It has been known for decades that some molecular clouds have obvious filamentary like structure. The recent
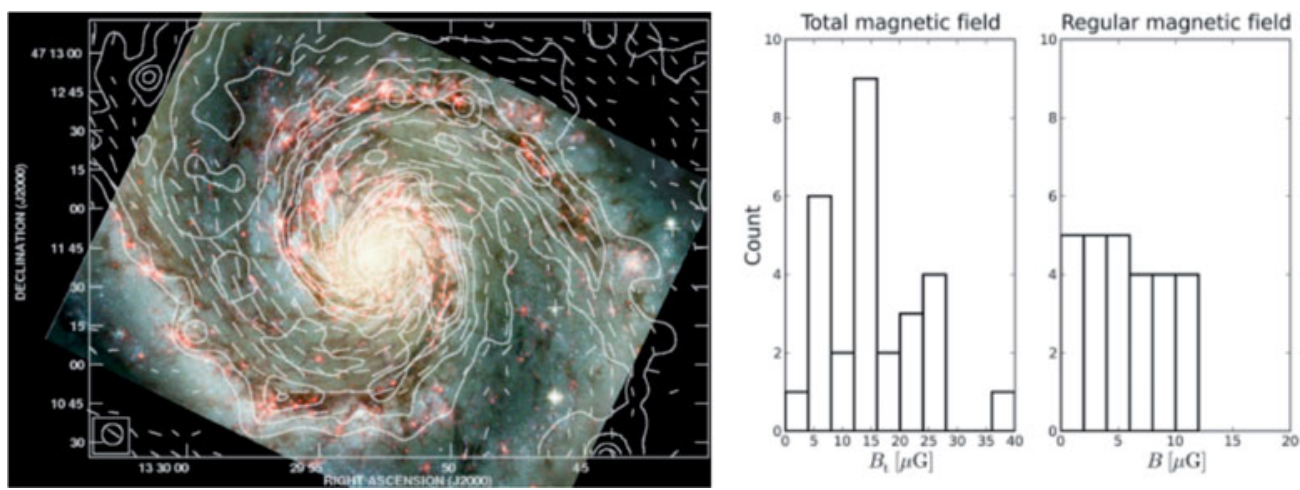

Figure 2. (a) Magnetic fields in the spiral galaxy M51. (b) Disitribution of the strengths of magnetic fields in spiral galaxies. Adapted from Fletcher et al. (2011). 
Herschel space observatory observations made a major breakthrough however by showing that filaments are ubiquitous - they occur on $10 \mathrm{pc}$ to less than $0.1 \mathrm{pc}$ scales in all molecular clouds (review, André et al. 2013, André et al. 2010, Menschchikov et al. 2011, Henning et al. 2011). Filamentary networks are seen in clouds without much evidence for star formation (eg. Polaris Flare, Ward-Thompson et al. 2010). In the Orion cloud, more than $70 \%$ of cores are strongly associated with filaments (Polychroni et al. 2013) indicating that the formation of filaments precedes star formation. Numerical simulations of supersonic turbulence in the interstellar medium have consistently shown that gas is compressed into filamentary networks (eg. Porter et al. 1994, Vasquez-Semadeni et al. 1994, Padoan et al. 2001). The addition of gravity in the simulations leads to the collapse of regions of denser gas to form stars (eg. Klessen \& Burkert 2000, Bonnell et al. 2003, MacLow \& Klessen 2004, Tilley \& Pudritz 2004).

The observations also reveal that star-forming cores are found preferentially in filaments that are gravitationally unstable. Equilibrium calculations show that filaments whose mass per unit length exceeds a critical value of $m_{c r i t}=2 c_{s}^{2} / G$ in purely thermally supposed gas ( $c_{s}$ is the sound speed) are gravitationally unstable (Inutsuka \& Miyama 1997). This condition is modified for magnetized filaments, or for turbulence where the condition becomes $m_{\text {crit }}=2 \sigma_{\text {tot }}^{2} / G$ for the total velocity dispersion including turbulence (Fiege \& Pudritz 2000). Magnetic fields can both thread filaments (poloidal component) providing additional pressure support against gravity, as well as wrap around filaments (toroidal component) which tends to squeeze them. The orientation of the magnetic field with respect to a filament in the Serpens molecular cloud is shown in Figure 3a. The observations of infrared ( $\mathrm{H}$ band) polarization of the cloud are overlaid on a column density map (Sugitani et al. 2011). The young Serpens South Cluster is at the intersection of a couple of filaments. The field strength is $\simeq 100 \mu G$ and its direction is generally perpendicular to the filament axis. This orientation of the field is also seen in other molecular clouds. Submillimeter polarimetry observations of cores in the Orion molecular cloud also tend to show fields that are perpendicular to the filament axis, as well as a general large scale ordered field (H-B Li et al. 2009). Optical polarization studies of the prominent filament in the Taurus B211 cloud shows a similar pattern (Palmeirim et al. 2012). A possible emerging trend in the magnetic field observations is that fields tend to to be perpendicular to filaments that are dense and forming stars, whereas they are aligned parallel to more diffuse filaments or fine striations. The latter are often found perpendicular to major filaments, and it is possible that the accretion flows onto filaments from the surrounding gas could be magnetically channeled. More measurements are required.
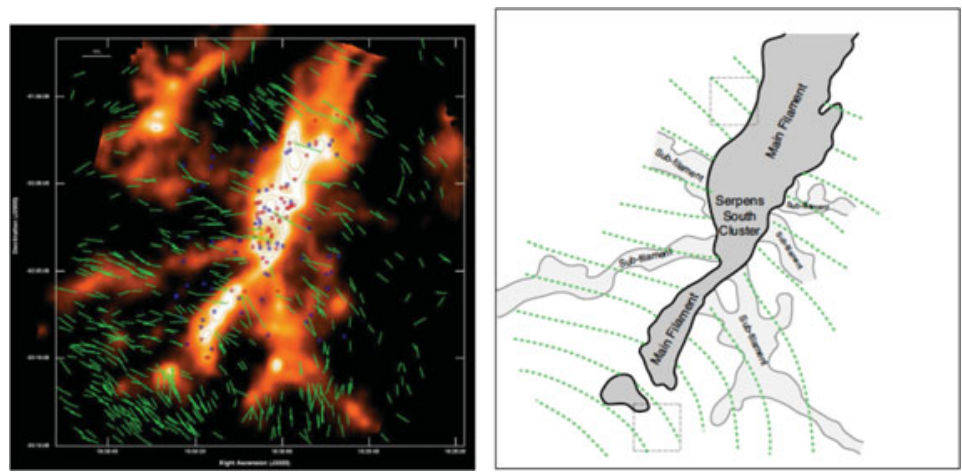

Figure 3. Orientation of the magnetic field with respect to the column density of gas in the filamentary cloud, Serpens-South in the Aquila complex. Adapted from Sugitani et al. (2011). 
Magnetic field strengths in molecular cloud cores are detected by Zeeman measurements (Crutcher et al. 2010, Crutcher 2012). These observations show that the magnetic field strength is a constant in the low density ISM up to $n \simeq 500 \mathrm{~cm}^{-3}$, and increases with density as $B_{\text {tot }, \text { max }}=10\left(\mathrm{n} / 300 \mathrm{~cm}^{-3}\right)^{0.65} \mu \mathrm{G}$, at higher densities characteristic of molecular gas. In molecular gas the field can be compressed. A measure of the importance of gravity relative to the magnetic energy in clouds is the mass to flux ratio, $\lambda=2 \pi \sqrt{G} \Sigma / B$ where $\Sigma$ is the column density of the gas and B is the field strength. The critical value, where gravitational density is comparable to the magnetic is $\lambda=1$. The observations show that there is an upper envelope for the data of $\lambda \simeq 2-3$ (Crutcher, 2012). At this level, simulations show that magnetic fields suppress the fragmentation of filaments (eg. Tilley \& Pudritz 2007).

We show, in Figure 4, the central 1 pc regions of two simulations of cluster forming clumps of mass $500 M_{\odot}$, early $(150,000 \mathrm{yrs})$ in the evolution of a self-gravitating turbulent cloud. On the left is a purely hydrodynamic simulation and an MHD simulation with $\lambda \simeq 2.33$ is on the right. The hydro filaments are more compressed and more apt to fragment. The MHD run has more diffuse filaments (due to magnetic pressure support) and somewhat less fragmentation (Kirk et al. 2014). Turbulence is raising the critical line mass threshold above the thermal value (contours red, blue, and white at 5 , 2, and 1 thermal critical line mass).

Simulations of supersonic turbulence have shown for nearly 2 decades that lognormal distributions of column density naturally arise. The reason is that shocks are a multiplicative process for building density fluctuations. In a medium that is repeatedly compressed by a random distribution of shock strengths, and in the limit of a large number of such events, a lognormal arises as a consequence of the central limit theorem (Padoan et al. 1997). Figure 5(a) shows that even a small number of shocks rapidly sets up a lognormal distribution in the density distribution (Kevlahan \& Pudritz 2009). In Figure 5(b) high resolution simulations without gravity produce a lognormal to high accuracy. Once gravity is turned on in this medium, a power law tail develops as the denser gas filaments start to be pulled together and ultimately collapse (Kritsuk et al. 2011), which agrees well with the observations (Kainulainen et al. 2009). Adding MHD to this does not change the results very much - there is only a weak dependence on the Alfvénic Mach number of
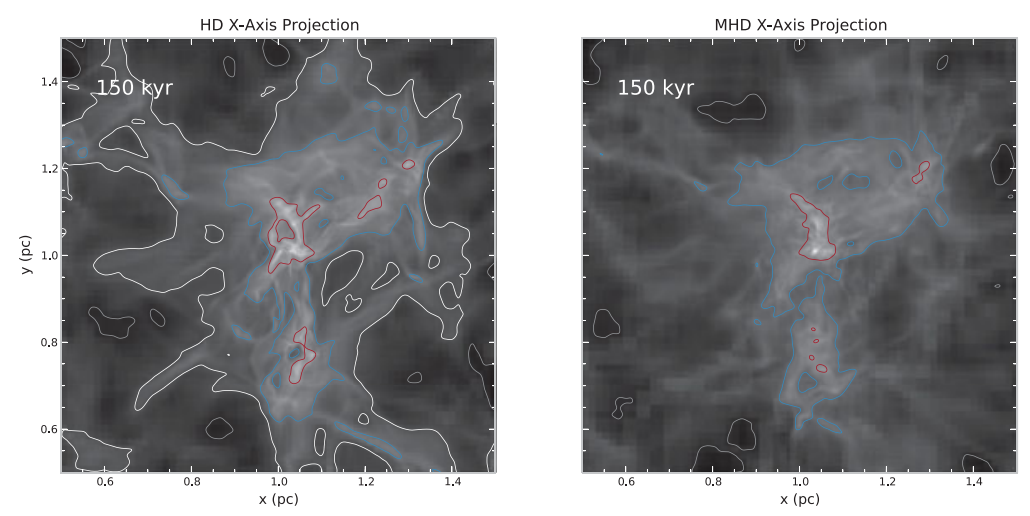

Figure 4. Filamentary structure: comparison of hydrodynamic and magnetohydrodynamic simulations of a turbulent, cluster forming clump. Column density maps for a $500 M_{\odot}$ cloud: hydro (left) and MHD (right) with $\lambda=2.33$. Red contour outlines 5 times $m_{\text {crit }}$ for the thermal critical line mass, blue is 2, and 1 is critical thermal line mass. Adapted from Kirk, Klassen, Pillsworth, and Pudritz (in prep). 
the hydromagnetic turbulence - the main effect being to broaden the effective half width of the lognormal (eg. Lemaster \& Stone 2008, Collins et al. 2011).

In summary, magnetic fields generated in galactic dynamos can clearly control the formation of molecular clouds, the dynamics of their filamentary structure, and the extent to which fragmentation into star forming cores takes place. There is a significant effect therefore on suppressing the formation of low mass cores. We now descend to much smaller scales, of $\leqslant 0.04 \mathrm{pc}$ and examine the collapse of cores, and the formation of disks and outflows.

\section{Magnetic Fields and the Origin of Protostellar Disks and Outflows}

Protostellar disks are observed around protostars of all masses - from brown dwarfs to massive stars. The angular momentum that is associated with molecular cloud cores arises from the collision of oblique shocks that produce the filaments (eg. Jappsen \& Klessen 2004, Banerjee et al. 2006). The specific angular momentum of cores on these $0.05 \mathrm{pc}$ scales is of the order $10^{21} \mathrm{~cm}^{2} \mathrm{~s}^{-1}$ which is 4 orders of magnitude greater than that of the young star that ultimately forms as a consequence of gravitational collapse. In purely hydrodynamic simulations, the collapse of such a region produces disks. The centrifugal radius that defines the outer edge of the disk under these conditions is typically $100 \mathrm{AU}$.

It has been known for some time that magnetic braking of rotating magnetized cores would easily occur (eg. Mouschovias \& Paleogolou 1980, Basu \& Mouschovias 1994). In the case of the gravitational collapse of a magnetized core, the faster rotating material deeper down in the collapsing region remains attached to the slower rotating material further out by the magnetic field, which therefore twists the field lines. The torsional Alfvén waves generated in this way carry off angular momentum, transferring it to the surrounding gas. What had not been anticipated was that in collapse calculations of cores threaded by ordered magnetic fields in clouds with $\lambda \leqslant 5$, the braking can be catastrophic, preventing the formation of rotationally supported disks (Mellon \& Li 2008, Hennebelle \& Fromang 2008, Duffin \& Pudritz 2009, Seifried et al. 2012a, Santos-Lima et al. 2012). The effect is not seen for sufficiently weak fields $(\lambda>10)$ but as noted, this does not fit the data for many cores.

Several solutions to this "magnetic braking catastrophe" have been suggested (see review Li et al. 2013) involving processes such as magnetic reconnection or lowered braking
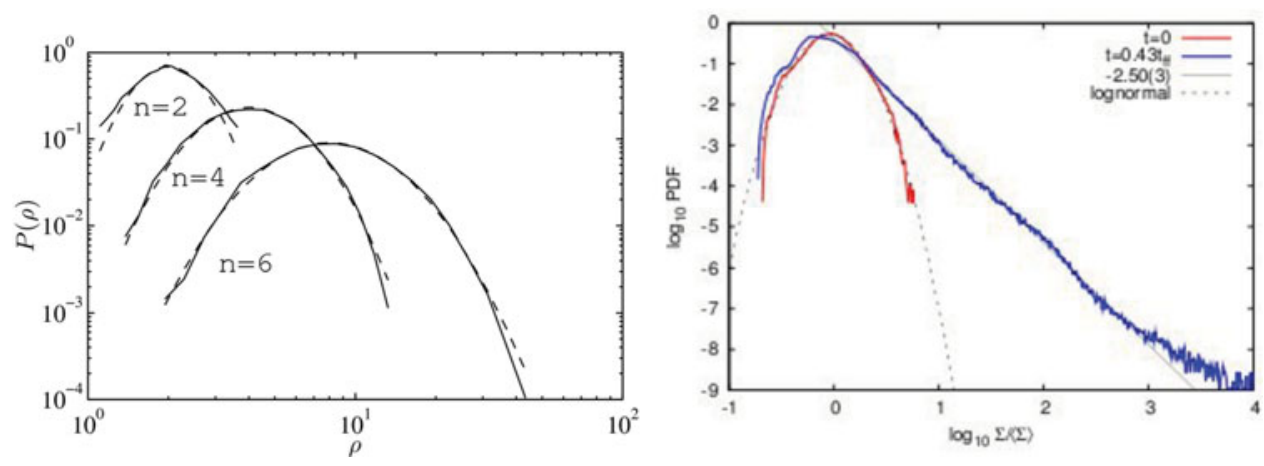

Figure 5. (a) Probability distribution function (PDF) of the density in a medium traversed by $\mathrm{n}$ shock waves. The dashed curves are best fit lognormal models. Adapted from Kevlahan \& Pudritz 2009. (b) Simulations of highly resolved 3D turbulence that without gravity, creates a lognormal PDF (red curve). When gravity is turned on, a power-law tail is generated (blue curve). Adapted from Kritsuk et al. (2011). 
efficiency by disks inclination to the mean field. Arguably the most robust solution to this problem is that turbulence facilitates the formation of rotationally supported disks (Seifried et al. 2012a - also Santos-Lima et al. 2012, Joos et al. 2013) . Figure 6(a) shows two simulation results for the formation of disks: the left figure shows that an ordered Keplerian disk does not form for ordered magnetic fields, while rotationally supported disks do form in the turbulent simulation (right). The rotation curves for disk-like structures in turbulent MHD collapse simulations are shown in Figure 6(b) for 4 different disks that form in an initially $100 M_{\odot}$ cloud simulations with $\lambda=2.6$ (Seifried et al. 2012a). In all cases, the rotation curves are Keplerian with much smaller radial inflow velocities.

The formation of magnetized accretion disks is accompanied by the launch of vigorous outflows. Outflows are among the earliest and most easily detectable signatures of star formation. This is well exemplified by the so-called Class 0 protostars in which much of the collapsing gas is still in the envelope and yet very energetic molecular outflows are present. Outflows can be driven by centrifugal acceleration (Blandford \& Payne 1982, Pudritz \& Norman 1983) or by the magnetic pressure gradient associated with the toroidal magnetic field, known as a tower flow (Lynden-Bell 1996, 2003). In a collapse, both types of outflow are observed in numerical simulations and there is now a vast literature on this subject featuring a wide range of numerical techniques (review, Pudritz et al. 2007, Li et al. 2013). Figure 7(a) shows the magnetic structure associated with both of these outflow types. The first is a slower tower flow that arises in the outer regions of forming disks and is likely associated with the formation of the first core (top frame). The second is the centrifugally driven wind from smaller disk radii (Banerjee \& Pudritz 2006).

The slow component is a transient outflow that in the later stages of disk evolution is dominated by the centrifugal disk wind. This is shown in Figure 7(b) for two different distributions of magnetic fields in the underlying disk (Staff et al. 2010). One notes that the dense material in the jet is confined more towards the central outflow axis, which is collimated by a strong toroidal field component that provides the collimating pinch force for the jet. This kind of jet structure is robust and does not depend on the details of how magnetic field is distributed in the disk. By applying the conservation laws of axisymmetric outflows, such as the generalized Bernoulli theorem (Pelletier \& Pudritz 1992), it is possible to show that both of these outflows are just different regimes of a single generalized acceleration condition that can nevertheless be successfully applied to dynamical outflow simulations (Seifried et al. 2012b).
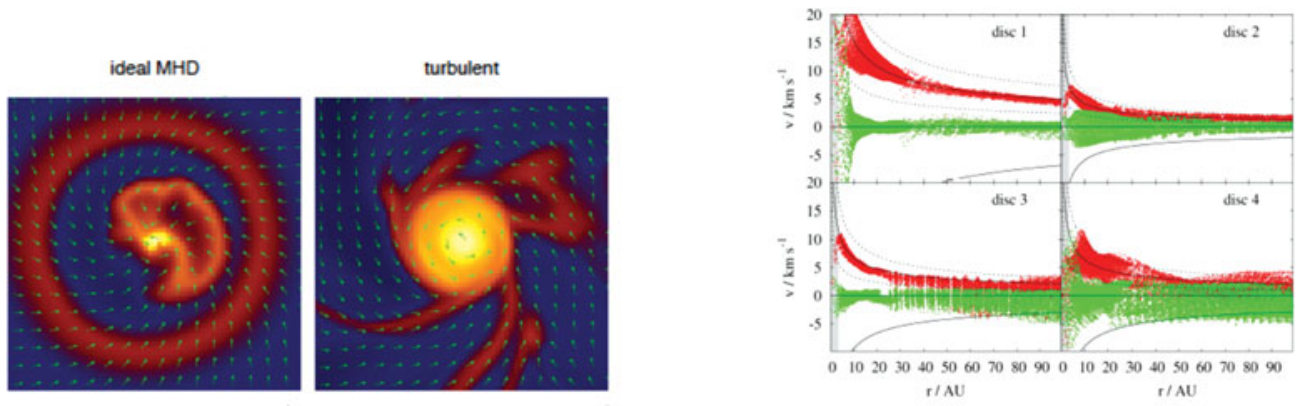

Figure 6. (a) Accretion of magnetized gas onto a central object in the absence (left) or presence (right) of turbulence. Adapted from Santos-Lima et al. (2012). (b) Rotation curves (red) and radial inflow speeds (green) through 4 different disks formed in 3D simulations of the collapse of magnetized turbulent, $100 M_{\odot}$ cloud. Solid line traces Keplerian rotation. Adapted from Seifried et al. (2012a). 
The importance of outflows for star formation is two fold. First, it is well known that MHD disk winds are highly efficient in extracting the angular momentum of disks - much more than viscous torques that arise through disk turbulence (Pelletier \& Pudritz 1992). HST observations suggest that jets may carry at least $60 \%$ of the angular momentum that must be shed by disks in order to enable accretion from disks onto their central stars (Baciotti et al. 2002, Anderson et al. 2005). The second important aspect of outflows is that they may be the agents that help disperse the collapsing gas in cores - giving rise to the conversion of the CMF to the IMF discussed in the introduction (Matzner \& McKee 2000). In more massive star formation, radiation fields will also play a significant role in core dispersal.

\section{Solving the Angular Momentum Problem for Young Stars}

We have now arrived at the magnetosphere of an accreting protostar as it truncates the accretion disk. The magnetic stresses associated with the star's magnetic field are strong enough at this radius $R_{t}$ to dominate the turbulent stress in the disk, so that matter free-falls along the field lines to the stellar surface. If the material at this last Keplerian orbital radius brings its angular momentum with it, the resulting spin-up accretion torque on the star is $\tau_{a}=\dot{J}=\dot{M}_{a} \sqrt{G M_{*} R_{t}}$, where $\dot{M}_{a}$ is the disk accretion rate. If unbalanced, this torque will spin up the star to break up speeds within $10^{5} \mathrm{yr}$. Several different solutions have been proposed for this problem. In the disk-locking picture of Königl (1991), dipolar field lines penetrating the disk beyond the co-rotation radius, $R>R_{c o} \simeq R_{t}$ would provide a spin-down torque. The difficulty with this idea is that the intense shear due to Kepler flow even a short distance from the co-rotation radius would create a very strong toroidal field, which would rapidly inflate and disconnect from the disk (Matt \& Pudritz 2004). Simulations of the interaction of a rotating magnetosphere inside an accretion disk confirm that stellar field lines penetrating near to the co-rotation radius undergo episodic inflation and ejection, much like coronal mass ejections on the Sun (Zanni \& Ferreira 2009).

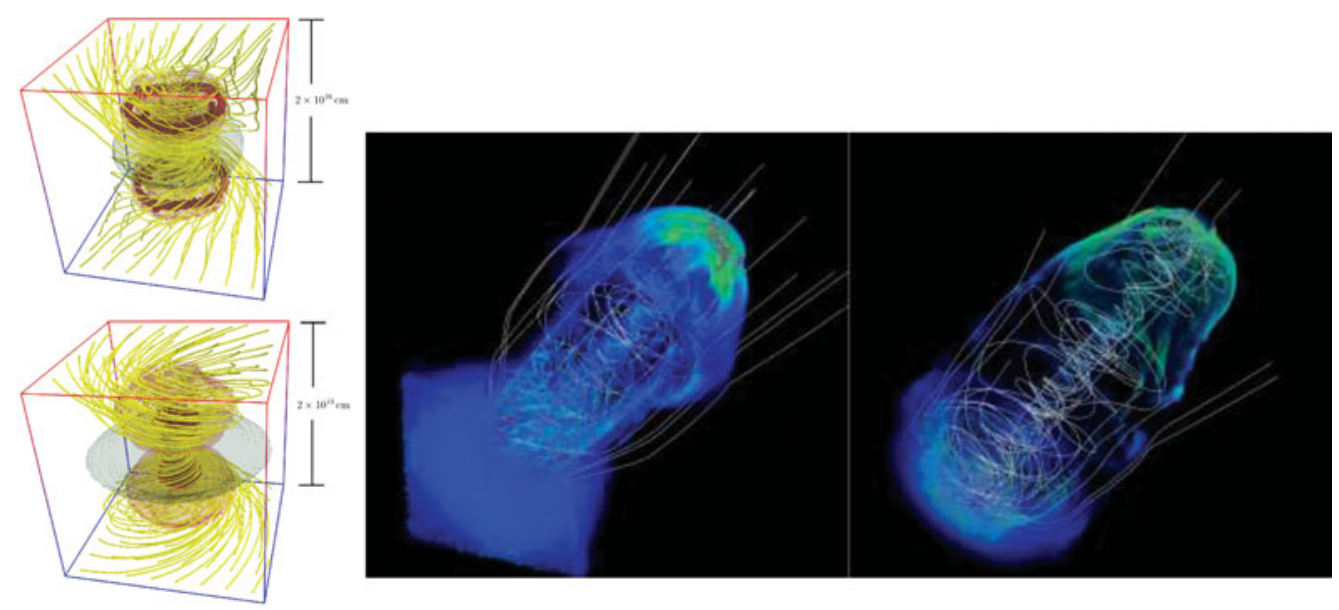

Figure 7. (a) Simulations of the collapse of a magnetized core; Top - magnetic field lines associated with a tower flow on 100 AU scales; Bottom - magnetic field lines associated with centrifugally driven disk wind from the inner region of the disk on $1 \mathrm{AU}$ scalpes. Adapted from Banerjee \& Pudritz (2006). (b) 3D simulations of jets from a Keplerian accretion disk for two different models for distribution of magnetic fields in the disk, including Blandford \& Payne (1982) and Ouyed \& Pudritz (1997). Adapted from Staff et al. (2010). 
An alternative idea is that the excess angular momentum is not deposited back into the disk, but is instead carried away by a strong magnetized acretion-powered stellar wind (Matt \& Pudritz 2005). The point is that the high accretion rates onto young stars liberate gravitational potential energy on impacting the photosphere. This powerful source of energy, if tapped at reasonable efficiencies, is available to drive a wind along the open magnetic field lines from the polar caps.

The four elements of a general theoretical framework involving both disk and stellar winds as well as magnetoshperic interaction are illustrated in Figure 8(a). At large disk radii beyond the interaction region is the disk wind. Magnetospheric accretion brings both gas and its angular momentum to the star where angular momentum is deposited and gravitational potential energy released. The third component is the accretion powered stellar wind carries off the accreted disk angular momentum. Finally, there is the interaction region between the stellar and disk winds. This interface between the disk and the magnetosphere is known from simulations to be highly time variable as accretion proceeds in bursts followed by the inflation of field lines (Goodson, 1999, Zanni \& Ferreira 2013). The accretion powered stellar wind (APSW) exerts a pressure on the surrounding disk wind, which prevents it from collimating as much as it might in its absence.

The spin down torque upon the star due to the APSW is $\tau_{w}=\dot{M}_{w} \Omega_{*} r_{A}^{2}$ where $r_{A}$ is the Alfvén radius of the stellar wind and $\dot{M}_{w}$ is the stellar wind mass loss rate. . In the theory of disk winds, the wind mass loss rate in disk wind theory is roughly a tenth of the observed accretion rate (Pudritz et al. 2007). The same kind of scaling is expected for APSW winds. Numerical calculations of 2D stellar magnetized winds assumed an initial dipole field and no disk or surrounding disk wind, for a variety of stellar magnetic field strengths, and found that for a $2 \mathrm{kG}$ field, a large Alfvén radius is obtained with $R_{A} \simeq 19.3 R_{*}$ (Matt \& Pudritz 2008). The fraction of the accretion power that is converted into wind power $(\epsilon)$ is one of the main parameters of the APSW theory. Equilibrium solutions for the slow spins can be found for various objects and this fixes the efficiency parameter. For a strong stellar field of $2 \mathrm{kG}$, the efficiency can be as low as $17 \%$ but this value must be higher for weaker dipole fields.

Our journey has now taken us to the accretion shock at the stellar photosphere. The question is what initiates the APSW? Thermal driving of the wind will not work because the cooling is highly efficient in the dense post-shock gas (Matt \& Pudritz 2008). A much less lossy mechanism of power conversion must be in play. One important possibility that
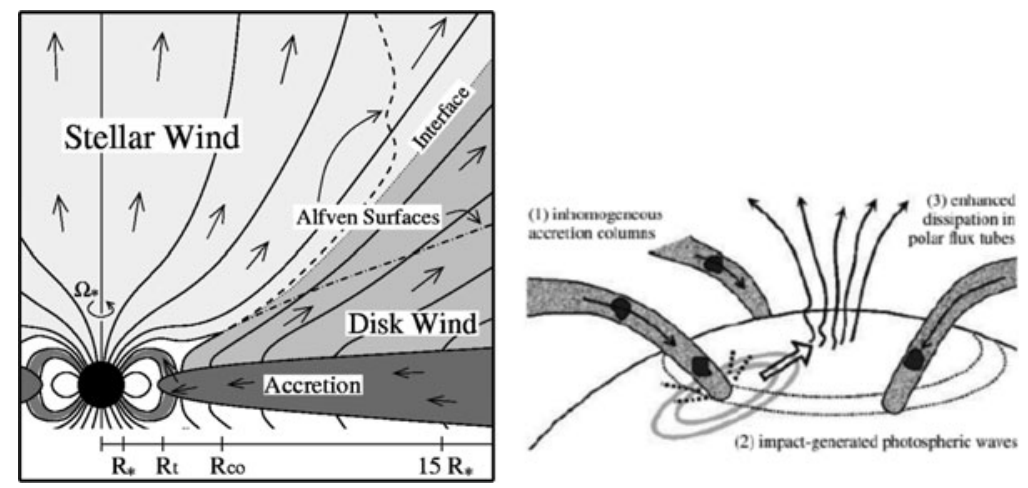

Figure 8. (a) A global view of disk winds, and accretion powered stellar winds associated with magnetized accretion disks and protostars. Adaped from Matt \& Pudritz (2005). (b) Illustration of the excitation of wave driven outflow by lumpy magnetospheric accretion onto the protostar. Adapted from Cranmer (2009). 
is the accretion power is converted to MHD wave driven winds (Matt \& Pudritz 2008(b). Wave driven stellar winds are well established for late type stars (Hartmann \& MacGregor 1980, DiCampli 1981). The shaking up of the stellar photosphere in the neighbourhood of the accretion column would launch waves propagating across the photosphere, as illustrated in Figure 8(b). Those regions with open magnetic field lines would be strongly excited leading to the launch of Alvén waves. The accretion column is likely to consist of lumpy gas. The energy released by each of these clumps in the inghomogenous accretion column is roughly $10 \%$ of the kinetic energy of the clump multiplied by the ratio of the clump density to the background flow. This scenario was worked out in some detail in two papers by Cranmer $(2008,2009)$ and found to be in rough agreement with observations although much more work remains to be done.

In conclusion, the role of magnetic fields in star formation is manifold and carries through many decades in physical scales. On the largest, magnetic fields can play a role in the formation and fragmentation of filamentary molecular clouds. The lognormal core of the IMF and CMF appears to be a direct consequence of turbulence, while gravity seems to be the dominant player in the creation of the high mass tail. Radiation and MHD together affect gravitational fragmentation and the CMF, with radiation dominating on smaller scales and MHD on larger. MHD turbulence, however, probably plays a dominant role in how disks are formed. Finally, angular momentum transport by magnetized winds and jets likely plays a central role in driving disk accretion and regulating the spins of young stars.

\section{References}

Alves, J., Lombardi, M., \& Lada, C. J. 2007, A\&A, 462, L17

Anderson, J. M., Li, Z. Y., Krasnopolsky, R., \& Blandford, R. D. ApJ, 630, 945

André, Ph., Men'shchikov, A., Bontemps, S., et al. 2010, A\&̈A, 518, L102

André, Ph., Men'shchikov, A., Könyves, V., \& Arzoumanian, D. 2011, in Computational Star Formation, IAU Symp. 270, Eds. J. Alves et al., p. 255

André Ph., Di Francesco J., Ward-Thompson D., Inutsuka, S-I., Pudritz, R. E., \& Pineda, J. 2014, in: Protostars and Planets VI, (Eds. H. Beuther, R. Klessen, K. Dullemond, \& T. Henning), in press.

Bacciotti, F., Ray, T. P., Mundt, R., et al. 2002, ApJ, 576, 222

Banerjee R. \& Pudritz R. E. 2006, ApJ, 641, 9491

Banerjee, R., Pudritz, R. E., \& Anderson, D. W., 2006, MNRAS, 373, 1091

Basu, S. \& Mouschovias, T. 1994, ApJ, 432, 720

Beck, R. 2005, in: Cosmic magnetic fields; Lecture Notes in Physics, 664, Eds. R. Wielebinski \& R. Beck, p. 41.

Beck, R. 2012, Space Sci Rev, 166, 215.

Beck et al. 1999,

Blandford, R. D. \& Payne, D. G. 1982, MNRAS, 199, 883

Bouvier, S. P. Matt, Mohanty, S., Scholz, A., Stassun, K. G., \& Zanni, C. 2014, in: Protostars and Planets VI, (Eds: H. Beuther, R. Klessen, K. Dullemond, \& T. Henning), in press.

Chabrier, G. 2005, PASP, 115, 763

Collins, D., Padoan, P., Norman, M. L., \& Xu, H. 2011, ApJ, 731, 59

Cranmer, S. R. 2006, ApJ, 689, 316

Cranmer, S. R. 2009, ApJ, 706, 824

Crutcher, R. M. 2012, ARA\&A, 50, 29

Crutcher, R. M., Wandelt, B., Heiles, C., Falgarone, E., \& Troland, T. H. 2010, ApJ, 725, 466

Decampli, W. M. 1981, ApJ, 244, 124

Duffin, D. F. \& Pudritz, R. E. 2009, ApJL, 706, L46

Elmegreen, B. G. 1982, ApJ, 253, 655

Fiege, J. D. \& Pudritz, R. E. 2000, MNRAS, 311, 85 
Fletcher, A. 2011, in The dynamic interstellar medium: a celebration of the Canadian Galactic Plane Survey, ASP Conference Series, 438, Eds. R. Kothes, T. L. Landecker \& A. G. Willis, 438, 197.

Fletcher, A., Beck, R., Shukurov, A., Berkhuijsen, E. M., \& Horellou, C. 2011, MNRAS, 412, 2396

Goodson, A. P. \& Winglee, R. M. 1999, ApJ, 524, 159

Hartmann, L. \& MacGregor, K. B. 1980, ApJ, 242, 260

Heesen, V., Krause, M., Beck, R., \& Dettmar, R.-J. 2009, A\&A, 506, 1123

Hennebelle, P. \& Fromang, S.2008, A\& $A, 477,9$

Henning, Th., Linz, H., Krause, O., et al. 2010, A\&A, 518, L95

Inutsuka, S. \& Miyama, S. M. 1997, ApJ, 480, 681

Jappsen, A.-K. \& Klessen, R. D. 2004, A\&BA, 423, 1

Johnstone, D., Wilson, C. D., Moriarty-Schieven, G., et al. 2000, ApJ, 545, 327

Joos, M., Hennebelle, P., \& Ciardi, A. 2012, A\&GA, 543, A128

Kainulainen, J., Beuther, H., Henning, T., \& Plume, R. 2009, A\&\&A, 508, L35

Kevlahan, N. \& Pudritz, R. E. 2009, ApJ, 702, 39

Kim, W.-T., Ostriker, E., \& Stone, J. M. 2003, ApJ, 595, 574

Kirk, H., Klassen, M., Pillsworth, S., \& Pudriz, R. E., 2014, in preparation.

Klessen, R. S. \& Burkert, A. 2000, ApJS, 128, 287

Königl, A. 1991, ApJ, 370, L39

Kritsuk, A., Norman, M. L., \& Wagner, R. 2011, ApJL, 727, L20

Lemaster, M. N. \& Stone, J. M. 2008, ApJ, 688, 905

Li, H.-b., Dowell, C. D., Goodman, A., Hildebrand, R., \& Novak, G., 2009, ApJ, 704, 891

Li Z-Y., Banerjee R., Pudritz R. E., Jorgensen J. K., Shang H., Krasnopolsky R., Maury A., 2014, in:Protostars and Planets VI, (edited by H. Beuther, R. Klessen, K. Dullemond, \& T. Henning ), in press.

Lynden-Bell, D. 1996, MNRAS, 279, 389

Lynden-Bell, D. 2003, MNRAS, 341, 1360

Matt, S. \& Pudritz, R. E. 2004, ApJL, 607, L43

Matt, S. \& Pudritz, R. E. 2005, ApJL, 632, L135

Matt, S. \& Pudritz, R. E. 2008, ApJ 681, 391

Matt, S., \& Pudritz, R. E. 2008, ApJ, 678, 1109

Matzner, C. D. \& McKee, C. F. 2000, ApJ, 545, 364

Mellon, R. R. \& Li, Z.-Y. 2008, ApJ, 681, 1356

Men'shchikov, A., André, Ph., Didelon, P., et al.2010, A\&GA, 518, L103

Motte, F., André, P., \& Neri, R.1998, A\&4A, 365, 440

Mouschovias, T. C. \& Paleologou, E. V. 1980, ApJ, 237, 877

Padoan, P., Nordlund, A., \& Jones, B. J. T. 1997, MNRAS, 288, 145

Padoan, P., Juvela, M., Goodman, A. A., \& Nordlund, A. 2001, ApJ, 553, 227

Pelletier, G. \& Pudritz, R. E. 1992, ApJ, 394, 117

Pudritz, R. E., Ouyed, R., Fendt, C., \& Brandenburg, A. 2007, in: Protostars and Planets V, (edited by B. Reipurth, D. Jewitt, and K. Keil), 277

Santos-Lima, R., de Gouveia Dal Pino, E. M., \& Lazarian, A. 2012, ApJ, 747, 21

Schneider, N., André, Ph., Könyves, V., et al. 2013, ApJL, 766, L17

Seifried, D., Banerjee, R., Pudritz, R. E., \& Klessen, R. S. 2012a, MNRAS, 423, L40

Seifried, D., Pudritz, R. E., Banerjee, R., Duffin, D., \& Klessen, R. S. 2012b, MNRAS, 422, 347

Sellwood, J. A. \& Balbus, S. A. 1999, ApJ, 511, 660

Staff, J. E., Niebergal, B. P., Ouyed, R., Pudritz, R. E., \& Cai, K. 2010, ApJ, 722, 1325

Sugitani, K., et al. 2011, ApJ , 734, 63

Ward-Thompson, D., Kirk, J. M., André, P. et al. 2010, A\&A, 518, L92

Tilley, D. A. \& Pudritz, R. E., 2004, MNRAS , 353, 769

Tilley, D. A. \& Pudritz, R. E., 2007, MNRAS, 382, 73

Zanni, C. \& Ferreira, J. 2009, A\&AA, 508, 1117

Zanni, C. \& Ferreira, J. 2013, A\&\&A, 550, 99 